\title{
Application of a Three-dimensional Printed Anatomical Model in Presurgical Planning of Surgical Treatment of Brain Aneurysms
}

\author{
Veranis Sotirios $^{1}$, Lagios Konstantinos ${ }^{2}$ \\ ${ }^{1}$ Neurosurgical Department, 251 General Air Force and Reserve Hospital, Athens, Greece \\ ${ }^{2}$ Neurointerventional Unit, 251 General Air Force and Reserve Hospital, Athens, Greece
}

Email address:

veranissotiris@gmail.com (V. Sotirios)

\section{To cite this article:}

Veranis Sotirios, Lagios Konstantinos. Application of a Three-dimensional Printed Anatomical Model in Presurgical Planning of Surgical Treatment of Brain Aneurysms. International Journal of Clinical and Developmental Anatomy. Vol. 5, No. 2, 2019, pp. 33-40. doi: $10.11648 /$ j.ijcda.20190502.13

Received: November 24, 2019; Accepted: December 20, 2019; Published: December 30, 2019

\begin{abstract}
Three dimensional (3D) printed models represent innovative tools in anatomy teaching and surgical planning. The present study aimed at generating 3D skull models incorporating middle cerebral artery (MCA) aneurysms and in assessing their anatomical accuracy and utility as training and preoperative planning tools. Two aneurysm models were generated. Initially, a full skull (model A) and subsequently a half skull (model B) using white polyactic acid (PLA) filament incorporating two arterial networks with hard black PLA filament (in model A) and a softer semitransparent filament (in model B). The models were based on computed tomographic angiography (CTA) of a female patient suffering from bilateral unruptured middle cerebral aneurysms. Model A, a high contrast model, was designed for anatomical illustration purposes. Model B was designed to allow for clipping simulations. The anatomical accuracy of the two models compared to CTA was assessed by measuring their dimensions at the neck, proximal, distal branches and fundus, using an electronic micrometer. The utility of the models for the comprehension of the underlying anatomy, pathology and preoperative planning was evaluated by means of online questionnaires following clipping simulations conducted by neurosurgery residents and specialized neurosurgeons. Of the two 3D printed models generated, model B (clipping model) showed the highest degree of anatomical accuracy. The results of the online survey on the utility of the proposed models indicate that the majority of participants accepted the innovation with positive responses and approve the use of 3D printed aneurysm models for preoperative planning and resident training.
\end{abstract}

Keywords: Three-dimensional Printing, Presurgical Planning, Brain Aneurysm, Aneurysm Clip, Middle Cerebral Artery Aneurysms

\section{Introduction}

Comprehension of surgical anatomy in the neurosurgery clinic is mainly accomplished by the analysis of Computed Tomography, CT, or Magnetic Resonance Imaging, MRI, data. Moreover, fusion of MRI, CT, PET images in virtual three dimensional, 3D, reconstructions of the underlying pathology by image editing software such as Stealth Medtronic, can provide the means for accurate presurgical planning [17]. In addition, there are training stations that utilize virtual reality techniques and haptics to simulate various operations $[7,20,25]$. Cadaveric studies provide the framework for training in anatomy and anatomic variations but they could not provide training for a specific pathology such as brain tumor neither they could permit accurate presurgical planning because the pathology is absent. 3Dprinted models of human anatomy represent innovative tools for anatomical education and preoperative or preinterventional planning $[20,11,21,26]$.

In this context, several 3D printed aneurysm models have been previously utilized in various applications that include not only preoperative planning and surgical resident training, but also during patient consultation [1-3]. Thus, Anderson et al [1] reported the 3D printing accuracy of 10 aneurysm 
models that were generated on the basis of Digital Subtraction Angiography, data. The accuracy of the replicas was assessed by quantifying statistical significance in the variations of their inner dimensions relative to 3D DSA images. These replicas could be used as flow phantoms in combination with phase contrast MRI. This means that injecting contract through the aneurysm replicas MRI flow studies and flow velocities inside the lumen of arteries harboring brain aneurysms could be feasible [1]. Kimura et al [2] studied 11 cases of brain aneurysms (3 retrospcective and 8 prospective) by making 3D hollow vessels models of the clipping site. Subsequently, used commercial titanium clips to evaluate accurate clipping positioning around the neck of the aneurysm [2]. They managed to confirm aneurysm's neck occlusion by using simultaneously an intraluminal endoscope. They transferred their findings to actual surgery (in 3 cases) and compared the $3 \mathrm{~d}$ model trial with the clip positioning during the operation. Each model costed 300-400 American Dollars and was created in 3-7 days [2]. Wurm et al published a method of cerebrovascular biomodeling for aneurysm surgery and used their models for aneurysm simulation surgery [3]. In their model, arterial network and cranial vault harboring a pterional craniotomy was printed based on rotational angiography 3D data [3]. Their model was accurate in dimensions and also calculated the tensile strength of the $3 \mathrm{D}$ printed vessels [3]. 3D printing process lasted 1.5 weeks and costed 2000 American dollars. They concluded that $3 \mathrm{D}$ printed models are a valuable education and training tool both for residents and experienced Neurosurgeons [3].

Khan et al reported prototyping of a paraclinod aneurysm model using 3D rotational angiography data [18]. Their model depicted anatomy in great accuracy and possible applications in research, training and surgical planning were suggested [18]. Enhancement of safety and reduction of surgical morbidity/mortality and fatal surgical errors by the introduction of 3D printed models in surgical practice has been suggested by Qiu et al [19]. Similar findings were reported by Nagassa et al [22]. Shibata et al published their technique on 3D-printing of aneurysm models based on CT data [9]. Their technique allowed for the generation of hollow vessel models suitable for hands on endovascular embolization trial [9]. They also reported concurrence relationship in size and shape between the model and the CT data [9]. Similar results have been published independently by Michael $\mathrm{W}$ et al [12]. Lin $\mathrm{J}$ et al reported 3D printing of cranial nerves emphasizing cranial base anatomy and applied these models in skull base tumor Surgery [14]. Van de Belt $\mathrm{TH}$ et al reported that $3 \mathrm{D}$ printed models are useful for patient education in Glioma Treatment [15]. Wang et al generated two different aneurysm models [10]. The first model had only the regional clipping site anatomy and the other one had the bone and proximal cranial nerves as well. They evaluated their model with a group of Neurosurgeons that made clipping simulation under the microscope. Efficiency of the models were evaluated with a questionnaire [10]. Lan Q et al reported the development of 3D printed
Craniocerebral models emphasizing the spatial relationship of the aneurysm to surrounding bony structures [8]. They used the model to simulate operation and surgical strategy in terms of contralateral feasibility of clip application and drilling necessity of bony structures such as anterior clinoid [8]. Benet et all made 3D printed brain aneurysm models and anastomosed the model with arteries of a prepared cadaveric skull and brain specimen to allow for more realistic training of clipping application [28].

The primary objective of the present study is to investigate how CT angiography, which is a common and rather quick and simple examination, could be utilized to make a $3 \mathrm{D}$ printed bio model of the arterial network regarding patients suffering from ruptured or unruptured brain aneurysms. Special attention was given to cost effectiveness and time interval between CT and actual model creation. The second objective was to evaluate the models by making a training event were a cohort of specialized and non-specialized doctors have had the opportunity to study the neurovascular anatomy and apply Sugita titanium clips trying to occlude the aneurysm.

To the best of my knowledge this is the first study in Greece assessing the feasibility of generating an anatomically accurate 3D printed aneurysm model and investigating its value in preoperative planning following clipping simulations conducted by doctors who were not involved in the process of making the model or delivering care to the patient. In addition to the arterial network was placed inside a complete bony cranium which gives a better spatial orientation and also permits craniotomy planning. This means that the final model incorporates not only the clipping site but the surrounding arteries, the skull base and the cranial vault as well. In the present study, two different models with distinct characteristics were created. One aimed at highlighting the neurovascular anatomy and the other for conducting clipping trials.

The patient selected for the generation of 3D printed models was chosen due to the fact that she had bilateral Middle Cerebral Artery, MCA, aneurysms and her MCA, aneurysms had a relative complex anatomy, a characteristic that would necessitate thorough anatomical understanding and would stimulate the decision-making process in the stage of presurgical planning. This type of bilateral MCA aneurysms according to one series are present in $14 \%$ of the patients harboring MCA aneurysms [16]. Their management is challenging and in most centers microsurgical approach is preferred over coiling. In addition to nowadays the majority of brain aneurysm are treated by coiling embolization and neurovascular techniques. MCA aneurysms are often complex in anatomy and surgical clipping or bypass techniques are preferred over coiling [29].

\section{Material and Methods}

\subsection{D Printed Model Preparation}

A 55 years old female patient with unruptured MCA 
aneurysms on both sides was selected from the 251 General Air Force and Reserve Hospital Neurovascular Database. Both patient and the Neurointerventional unit of the hospital consented to release the data for further anonymous utilization [23]. The patient was admitted on the 3 of July 2017 with symptoms of sudden onset headache. Bilateral MCA aneurysms were diagnosed with CTA and DSA. Left MCA aneurysm had a complex configuration. It was located at the left M1 bifurcation and its length was $20 \mathrm{~mm}$ with a dome to neck ratio $>1$. Furthermore, aneurysm was adherent to both M1 branches and an inferior temporal M2 branch at the level of the neck of the aneurysm. Right MCA aneurysm was located at the M1 bifurcation and had a smaller size ( $7 \mathrm{~mm}$ ) with dome to neck ratio $<1$ (Figure 1). The aneurysms were unruptured and no subarachnoid blood was detected with head CT scan and Cerebrospinal Fluid, CSF, examination. The patient was treated with coil embolization of both the aneurysms without complications. DICOM (Digital Imaging and Communications in Medicine) images of the CT - angiography dataset (images 1-4) were selected and transformed to an NRRD (Nearly Raw Raster Data) file which permits further transformation and is devoid of patient personal information. DICOM is the international standard to transmit, store, retrieve, print, process, and display medical imaging information [5]. The DICOM images dataset of the patient was produced and obtained directly from the CT scanner were the CTA was performed. The DICOM folder contains multiple pictures each of them contains metadata regarding patient personal information. NRRD file is a single file containing all the images of the patient, without any personal information and could be easily used for volume rendering. The NRRD file was made through Slicer $3 \mathrm{~d}$ open source software from the DICOM upload command and then saving the study using. nrrd suffix. The NRRD acronym means, nearly raw raster data, and it is a library and file format for the representation and processing of $n$ dimensional raster data. It is intended use is to support scientific visualization and image processing applications [6].

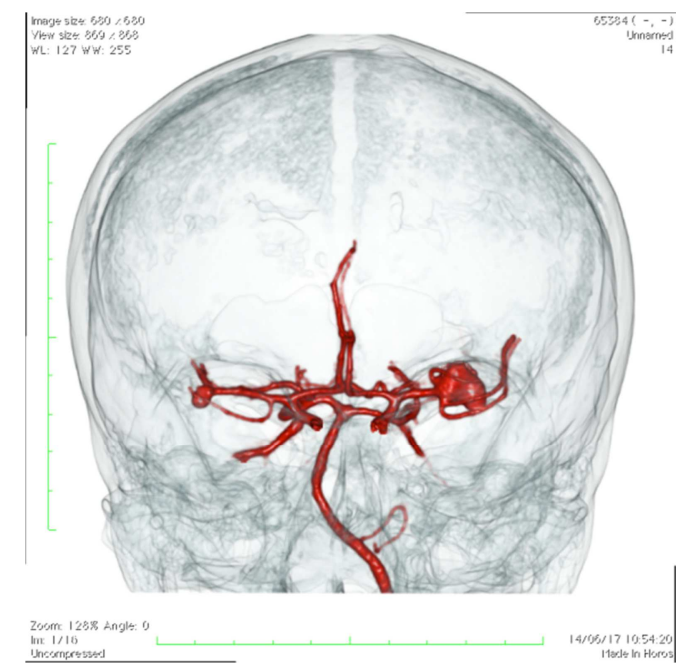

Figure 1. $3 D$ reconstruction of the CTA showing Right, and Left MCA aneurysms, notice the bifurcation of M1 (blue arrows) and temporal M2 cortical branches.
Slicer 3d open source software has been used for volume segmentation and $3 \mathrm{~d}$ object formation. Volume segmentation was performed by using threshold from 170-1500 Hounsfield units. As a result, a 3D- volume that contained the anatomy of the skull and the arterial network was produced. Next step involved saving the work at an STL file format. The STL archive was further refined with Mesh mixer freeware software and the final code send to a 3D printer for printing. STL is anabbreviation of "stereolithography" and it is a file format native to the stereolithographysoftware created by $3 \mathrm{D}$ Systems [4].

Two different models were printed with half skull (model B) and full skull (model A) using white PLA filament and two arterial networks with hard black PLA (polyactic acid) filament (model A) and a softer semitransparent (Embodi3D) filament (model B). Both models were printed by Embodi3D which is based near Seattle, Washington, USA.

The cost for the first model was 170 euros and for Model $\mathrm{B}$ was 250 euros. Average manufacture and shipping interval from the online order was 20 days. Final results are depicted in Figures 2 and 3.

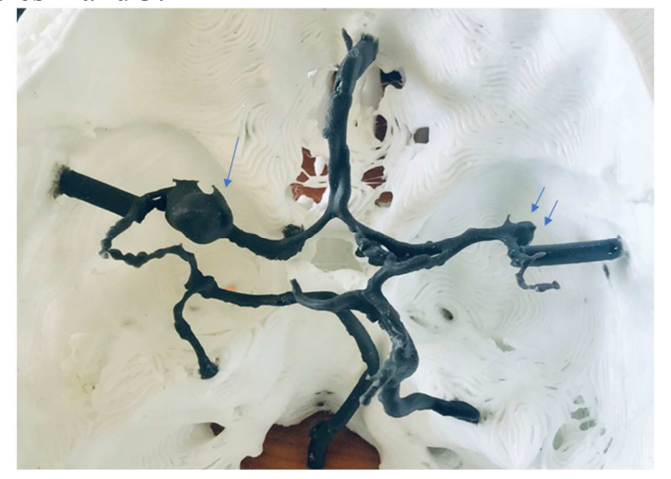

Figure 2. Model A depicts circle of Willis and a complex left MCA (single arrow) and right MCA, (double arrows) aneurysms.

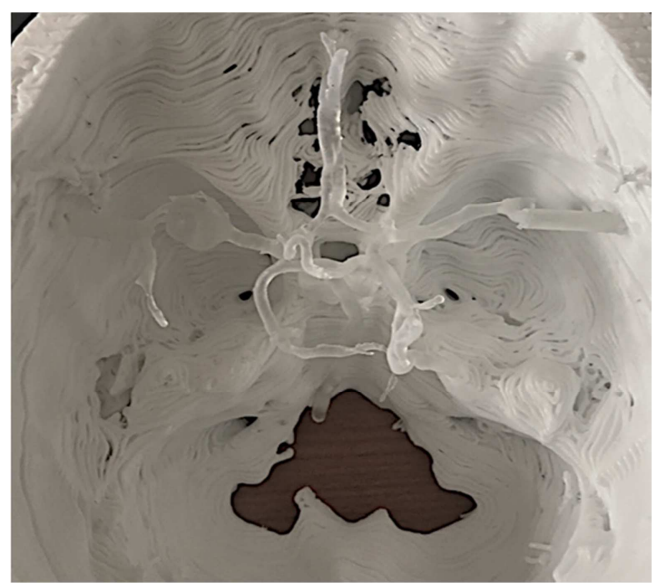

Figure 3. Model B from softer semitransparent plastic depicts left (single arrow) and right MCA, (double arrows) aneurysms, red arrows depict veins.

\subsection{Aneurysm Clipping Simulations and the Evaluation of 3D Models in Presurgical Planning Using Online Surveys}

The final models of 3D printing were evaluated by a group of 13 doctors with mixed levels of specialization. Following 
analysis of the CTA and DSA clinical details, trial clipping of both aneurysms using Sugita clips was performed by the enrolled physicians. A left or right pterional craniotomy accordingly was assumed. There was also 20-minute time given for anatomy comprehension and spatial orientation of the arterial web. Another half an hour interval was given for each participant to apply various Sugita clips around the neck of the aneurysms. Conversation was enhanced between the more experienced and the less experienced physicians to assess feasibility of aneurysm clipping and complications avoidance. Participants had also access to Virtual 3D Models generated with OSIRIX software. OSIRIX is made by Pixmeo SARL, which is a Swiss company, based in the Geneva area.

After the hands-on experience and multiple clipping trials of the aneurysms using various Sugita clips, there was a brief survey taken by each using Survey Monkey Inc. application. Survey Monkey is an online cloud-based software company specialized un questionnaire development and analysis and its headquarters are based in San Mateo California, USA.

A survey was send individually to participant's email addresses and results recorded and stored unanimously. The survey questions (Table 2, Appendix A) allowed for the assessment of the age and experience of the participants and of the 3D model's utility in improving neurovascular anatomy comprehension and in aiding preoperative planning. A final general question investigated how the cohort felt about incorporating $3 \mathrm{D}$ printed surgical models in practice.

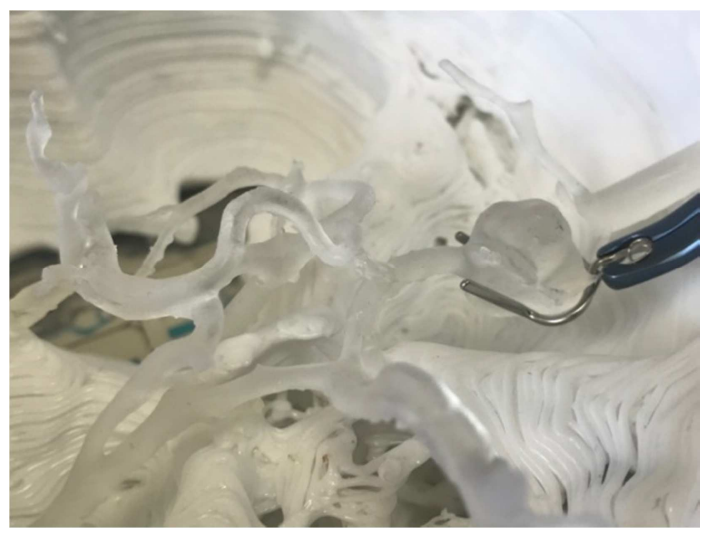

Figure 4. Clipping trial of the left aneurysm with a right angle fenestrated clip. Noticed that clip remains open simulating the hardness of the aneurysm sack because of atherosclerosis.

\section{Results}

\subsection{D-printed Models and CTA Comparisons}

Representative photographs of the two aneurysm models are shown in Figures 2, 3 and 4. The two 3D printed models were compared with Computed Tomographic Angiography, CTA, data to assess anatomical accuracy. Aneurysm dimensions were measured using an electronic micrometer, OEM ltd. at the neck, proximal, distal branches and fundus. Left MCA was divided in two branches that are mentioned as frontal and temporal branches, table 1. Measurements were compared with CTA data, table 1.

Table 1. Comparison of $3 D$ Models with CTA DATA.

\begin{tabular}{|c|c|c|c|c|c|}
\hline ANATOMICAL PARAMETERS & MODEL A mm & MODEL B mm & CTA mm & $\begin{array}{l}\text { Model A-CTA } \\
\text { difference mm }\end{array}$ & $\begin{array}{l}\text { Model B- CTA } \\
\text { difference mm }\end{array}$ \\
\hline M1 RIGHT WIDTH & 2,94 & 3,14 & 2,38 & 0,56 & 0,76 \\
\hline M1 LEFT WIDTH & 3,15 & 3,25 & 2,22 & 0,93 & 1,03 \\
\hline M2 LEFT FRONTAL BRANCH WIDTH & 1,94 & 1,9 & 1,7 & 0,24 & 0,2 \\
\hline $\begin{array}{l}\text { M2 LEFT TEMPORAL BRANCH } \\
\text { WIDTH }\end{array}$ & 1,84 & 1,50 & 1,40 & 0,44 & 0,1 \\
\hline LEFT ANEURYSM LENGTH & 14,22 & 14,6 & 15,00 & 0,78 & 0,4 \\
\hline M2 RIGHT WIDTH & 2,09 & 1,92 & 1,0 & 1,09 & 0,92 \\
\hline RIGHT ANEURYSM LENGTH & 7,97 & 5,61 & 5,30 & 2,67 & 0,31 \\
\hline RIGHT ANEURYSM WIDTH & 5,08 & 5,04 & 4,10 & 0,98 & 0,94 \\
\hline
\end{tabular}

Comparing both models with CTA and using a cutoff of $1 \mathrm{~mm}$ as an accuracy margin, the following points can be made: Model A was accurate in 7 out of 9 measurements and model B in 7 out of 9 measurements. Model A was not accurate in aneurysm length by $2,67 \mathrm{~mm}$. Model B was by $1,21 \mathrm{~mm}$ smaller in aneurysm sac width. Overall Model B had smaller differences compared to CTA than model A. Overall, in comparison to CTA findings, Model B displayed smaller margins of error in the majority of measurements ( 6 out of 9 for model B vs 4 out of 9 for model A).

\subsection{Online Survey Findings}

There were 13 participants in clipping simulations, 13 online responses to the survey and 11 complete responses. Completion rate was 85\%. Last Entry was in 03/07/2019.
There were nine questions. Most participants found that 3D models were extremely useful in understanding the underlying anatomy of the pathology. $76,92 \%$ of the participant found that $3 \mathrm{~d}$ model clipping experience was extremely useful in making a preoperative plan. In question 3 , although not having a Neurointerventional experience, $64 \%$ of the participants felt that $3 \mathrm{~d}$ models would have been helpful in making a coiling planning. The level of experience in treating patients with brain aneurysms was assessed. Experience either as a surgeon or as a resident or clinical assistant in managing patients suffering from brain aneurysms was noted and $9.69,23 \%$ of the participants found that the models were very accurate compared to CTA both in dimensions and spatial orientation. $81,82 \%$ of the participants agreed that incorporating $3 \mathrm{~d}$ models in clinical practice 
would be helpful in presurgical planning. $90.91 \%$ of the participants reported that $3 \mathrm{~d}$ models were more helpful in understanding bilateral aneurysm anatomy, especially on the left side, compared to virtual $3 \mathrm{~d}$ model based on the CTA.

In practice, it was clear that the left complex aneurysm would not be an easy case intraoperatively due to the difficulties in placing the clip without interfering with M2 branches. One of the techniques proposed to assess this problem was to cut and remodel the sac after temporal M1 clipping.

\section{Discussion}

Printing a model in order to depict surgical anatomy presents numerous technical difficulties. In the present study, the aneurysm patient chosen for the generation of the $3 \mathrm{D}$ models, harbored bilateral MCA aneurysms. The left MCA bifurcation aneurysm was complex with both temporal and frontal branches adherent to the aneurysms' sac. The models used for this study were harder than brain vessels. Both structures were compact and this prevented total collapse of the neck of the aneurysm.

In the present study, two different models were generated, serving different purposes, models A and model B. Model A was designed for neurovascular anatomy illustration purposes Thus, a black PLA thread was used to achieve maximum contrast with the white skull base. One of the disadvantages of this model was that hardness of the material prevented aneurysm clip closure. This was the main reason that a second model was designed and created. Model B had the same white PLA used to make the skull base but the arterial network was made with a soft semitransparent thread that was compressible to simulate better aneurysm clipping. Both three dimensional printed models were compared with CTA data. Models were measured with an electronic micrometer with an accuracy of 1/100 of mm. CTA measurements were made with the length cursor in OsiriX environment. A difference of more than $1 \mathrm{~mm}$, compared to CTA data was considered inaccurate [27]. This assumption was based on the fact that actual surgery dimensions of the arterial networks incorporate the arterial wall in contrast to the intraluminal dimensions represented by the angiography. Model A was by $2,67 \mathrm{~mm}$ larger in aneurysm length compared to CTA. This fact may have been caused by the printing process because there was a harder PLA thread used for printing of model A. Model B had a difference of $1,21 \mathrm{~mm}$ in left aneurysm's sac width. Overall model B had smaller differences compared to the CTA. This could be explained by the different softer filament used for making model $\mathrm{B}$ which permits more accurate printing.

There are studies that generated hollow 3D printed vessels. $3 \mathrm{D}$ printed vessels with a lumen are more accurate in aneurysm clipping simulations because the walls of the aneurysm are compressible and the clip can be applied and closed around the neck of the aneurysm. Nevertheless, the technical expertise needed to make that are more complex and more expensive. [12-13].

One of the challenges in making 3D models for neurosurgical planning is to match the spatial relationship of the pathology with the brain and cranial nerves and address issues such as elasticity and hardness of the material compared with the real anatomy. Wang L et al [10] and Lan $\mathrm{Q}$ [8] used three dimensional printed models incorporating the anatomy of the skull base and cranial nerves in a more detailed manner. It should be mentioned that as complexity of the model increases so as the time needed for the production of the model and its cost $[10,8]$.

Vessels are hollow anatomical structures. Other researchers as Michael W [12] et al and Mashiko T [13] have managed to print hollow vessels. These models were used in simulating endovascular techniques although the length of the catheterized vessels was very short compared to the actual procedure [12-13]. In addition to three-dimensional printed hollow vessel models could simulate clipping more faithfully since there is a lumen that could be clipped at the level of the aneurysm neck [13].

Evaluation of both models was carried out using a questionnaire of 9 questions focusing on how educative and suitable for preoperative planning the models could be. Questions were divided in three groups. Questions 1,5,6 focused on the educative role of the 3D printed models. Questions 2,3,7 were relevant to presurgical or preinterventional planning. Q 4,8,9 were targeted on defining the level of the expertise of each of the participants. In Q9 "How often do you treat patients with brain aneurysm" the purpose was to reveal how often the participant was exposed to aneurysm treatment as a member of a team and not actually the leading surgeon or neurovascular interventionist.

Considering the first group of questions 1,5, and 6 the results of the questionnaires indicate that $100 \%$ of the participants found the addition of three dimensional printed models useful (Question 1: extremely useful + very useful $=100 \%$ ). This result indicate that surgeons and residents are eager to have any extra tool for making a plan before going to operating theatre which is in accordance to modern neurosurgical planning. In question 5 the majority of the participants $(69 \%)$ found the $3 \mathrm{D}$ models accurate. It is worth mentioning that accuracy was subjectively reported by in terms of relevance to actual shape, size and spatial orientation. The main result from this observation is that surgeons tend to make a mental image of the pathology and compare other input data from CTA, 3D printed models with this image. In question 6 most of the participants 90,91\% reported that $3 \mathrm{D}$ models were better for anatomy understanding compared to CTA. A very important conclusion could be extracted from this result and which is that when haptic stimulations are included, understanding is improved.

In the second group of questions assessment of the value of the model in presurgical planning was attempted. In question $2,76 \%$ reported that $3 \mathrm{D}$ models were useful in presurgical planning of a coiling procedure. It is interesting to note that in question 3,60\% reported that playing around with the 3D models would have been helpful in planning a coiling procedure even though none of the participants was an experienced interventionist. These results suggest that interference with the neurovascular anatomy in the three 
dimensions increases the level of confidence. On the other hand, limitations to this confidence increase effect are obviously present. This means that although training and preoperative planning with $3 \mathrm{D}$ models obviously enhances neurovascular anatomy understanding, this cannot totally substitute actual supervised surgical experience. In Question 7, $80 \%$ of the participants would like to incorporate $3 \mathrm{D}$ models in their practice as a training adjunct. This result is absolutely meaningful given that modern surgeons feel confident to carry out an operation when they accumulate theoretical knowledge, practical experience under supervision, cadaveric laboratory experience and preoperative imaging studies. 3D printed models of the surgical pathology was treated as one more tool towards surgical success.

The third group of questions investigated experience of the cohort. Age is a general marker of experience if continuous surgical activity is granted. Only one of the participants was over 55 years old and one over 45 . The group participants were therefore a rather inexperienced group in aneurysm clipping surgery. It is reasonable to assume that the inexperienced surgeon is eager for any training tool that would speed up their learning curve. The same conclusion can be reached on the basis of the results to questions 9 and 4 that confirmed that the level of actual surgical experience of the participants was low. Experienced neurovascular surgeons clip 3-4 aneurysms per month which is added to approximately 30-40 aneurysms per year. In the institution were the study was conducted and according to medical files the number of brain aneurysm that is treated with coiling is 40 per year approximately in contrast to the number of aneurysm that is clipped which is approximately 2 per year.

As a future direction, more complex questionnaires and involvement of the participants in making measurements and of both length and elasticity of the models should be more accurate for evaluation purposes. Ideally there should be prospective trials of evaluating the presurgical planning, that has been made using three dimensional printed vascular models, with intraoperative anatomy of the same aneurysms. In other studies evaluation of the models was rather empirical [1-4]. Another future direction is that to better simulate surgery more complex 3D printed models should be generated and incorporate vascular, bony and brain anatomy in the same model. Moreover, hollow arterial networks filled with red colored liquid, flowing inside the vessels, will give a more realistic perspective [24].

\section{Conclusion}

The present study supports the feasibility of generating anatomically accurate 3D printed aneurysm models based on CTA data. Production time from examination to model delivery and cost were reasonable and could be improved by setting up industrial class $3 \mathrm{D}$ printers at the vicinity of hospital. The results of the online survey indicate that the participating neurosurgeons accepted the innovation with positive responses and favored the use of the models in preoperative planning and resident training.

\section{Abbreviations}

MCA middle cerebral artery, 3D, three dimensional, PLA, polyactic acid, NRRD near row raster data, DICOM Digital Imaging and Communications in Medicine, CT computed tomography, MRI magnetic resonance imaging, DSA digital subtraction angiography, STL stereolithographic, CTA computed tomographic angiography, PET positron emission tomography.

\section{Appendix}

Table 2. Assessment Questionnaire.

\begin{tabular}{ll}
\hline Q1 & $\begin{array}{l}\text { How useful is the 3D model in understanding the underlying } \\
\text { anatomy of the patient? }\end{array}$ \\
Q2 & $\begin{array}{l}\text { How useful is the model in making a preoperative plan for } \\
\text { clipping the aneurysm? }\end{array}$ \\
Q3 & How useful is the model to plan a coiling procedure? \\
Q4 & What is the level of your experience? \\
Q5 & What is the level of accuracy of the model compared to the CTA? \\
Q6 & What helped you the most for defining the aneurysm anatomy? \\
Q7 & What do you think about incorporating 3D printed models in \\
Q8 & Surgical preoperative planning? \\
Q9 & How often do you treat patients with brain aneurysms? \\
\hline
\end{tabular}

\section{References}

[1] Anderson J R, Thompson W L, Alkattan A K, Diaz O, Klucznik R, et al. 2016. Three-dimensional printing of anatomically accurate, patient specific intracranial aneurysm models J. Neurointerv. Surg. 8: 517-20.

[2] Kimura T, Morita A, Nishimura K, Aiyama H, Itoh H, Fukaya S, Sora S, Ochiai C. Simulation of and training for cerebral aneurysm clipping with 3-dimensional models. Neurosurgery. 2009 Oct; 65 (4): 719-25; discussion 725-6. doi: 10.1227/01.NEU.0000354350.88899.07.

[3] Gabriele Wurm, MD, Michael Lehner, MD, Berndt Tomancok, MD, Raimund Kleiser, PhD, and Karin Nussbaumer, MD Cerebrovascular Biomodeling for Aneurysm Surgery: Simulation-Based Training by Means of Rapid Prototyping Technologies Surgical Innovation 18 (3) 294-306, 2011.

[4] Chua, C. K; Leong, K. F.; Lim, C. S. (2003), Rapid Prototyping: Principles and Applications (2nd ed.), World Scientific Publishing Co, ISBN 981-238-117-1 Chapter 6, Rapid Prototyping Formats.

[5] Kahn, Charles E.; Carrino, John A.; Flynn, Michael J.; Peck, Donald J.; Horii, Steven C. (September 2007). "DICOM and Radiology: Past, Present, and Future". Journal of the American College of Radiology. 4 (9): 652-657. doi: 10.1016/j.jacr.2007.06.004.

[6] Aja-Fernandez, Santiago; de Luis Garcia, Rodrigo; Tao, Dacheng; Li, Xuelong (2009). Tensors in Image Processing and Computer Vision. Advances in Computer Vision and Pattern Recognition. Springer Science \& Business Media. ISBN 9781848822993. 
[7] Ghasemloonia A, Baxandall S, Zareinia K, Lui JT, Dort JC, Sutherland GR, Chan S. Evaluation of haptic interfaces for simulation of drill vibration in virtual temporal bone surgery. Comput Biol Med. 2016 Nov 1; 78: 9-17. doi: 10.1016/j.compbiomed.2016.09.005. Epub 2016 Sep 12. PubMed PMID: 27643462.

[8] Lan Q, Chen A, Zhang T, Li G, Zhu Q, Fan X, Ma C, Xu T. Development of Three-Dimensional Printed Craniocerebral Models for Simulated Neurosurgery. World Neurosurg. 2016 Jul; 91: 434-42. doi: 10.1016/j.wneu.2016.04.069. Epub 2016 Apr 27. PubMed PMID: 27132180.

[9] Shibata E, Takao H, Amemiya S, Ohtomo K. 3D-Printed Visceral Aneurysm Models Based on CT Data for Simulations of Endovascular Embolization: Evaluation of Size and Shape Accuracy. AJR Am J Roentgenol. 2017 Aug; 209 (2): 243-247. doi: 10.2214/AJR.16.17694.

[10] Wang L, Ye X, Hao Q, Chen Y, Chen X, Wang H, Wang R, Zhao Y, Zhao J. Comparison of Two Three-Dimensional Printed Models of Complex Intracranial Aneurysms for Surgical Simulation. World Neurosurg. 2017 Jul; 103: 671679. doi: 10.1016/j.wneu.2017.04.098. Epub 2017 Apr 24.

[11] Ploch CC, Mansi CSSA, Jayamohan J, Kuhl E. Using 3D Printing to Create Personalized Brain Models for Neurosurgical Training and Preoperative Planning. World Neurosurg. 2016 Jun; 90: 668-674. doi: 10.1016/j.wneu.2016.02.081. Epub 2016 Feb 24. PubMed PMID: 26924117.

[12] Michael W. Itagaki Using 3D printed models for planning and guidance during endovascular intervention: a technical advance Diagn Interv Radiol. 2015 Jul-Aug; 21 (4): 338-341. Published online 2015 May 29. doi: 10.5152/dir.2015.14469.

[13] Mashiko T, Otani K, Kawano R, Konno T, Kaneko N, Ito Y, Watanabe E. Development of three-dimensional hollow elastic model for cerebral aneurysm clipping simulation enabling rapid and low cost prototyping World Neurosurg. 2015 Mar; 83 (3): 351-61. doi: 10.1016/j.wneu.2013.10.032. Epub 2013 Oct 16.

[14] Lin J, Zhou Z, Guan J), Zhu Y), Liu Y, Yang Z, Lin B, JiangY, Quan X, Ke Y, Xu T. Using Three-Dimensional Printing to Create Individualized Cranial Nerve Models for Skull Base Tumor Surgery World Neurosurg. 2018 Dec; 120: e142-e152. doi: 10.1016/j.wneu.2018.07.236. Epub2018 Aug 16.

[15] van de Belt TH, Nijmeijer H, Grim D, Engelen LJLPG, Vreeken R, van Gelder MMHJ, Ter Laan M. Patient-Specific Actual-Size Three-Dimensional Printed Models for Patient Education in Glioma Treatment: First Experiences. World Neurosurg. 2018 Sep; 117: e99-e105. doi: 10.1016/j.wneu.2018.05.190. Epub 2018 Jun 2. PubMed PMID: 29870846.

[16] Elsharkawy A, Lehečka M, Niemelä M, Billon-Grand R, Lehto H, Kivisaari R, Hernesniemi J. A new, more accurate classification of middle cerebral artery aneurysms: computed tomography angiographic study of 1,009 consecutive cases with 1,309 middle cerebral artery aneurysms. Neurosurgery. 2013 Jul; 73 (1): 94-102; discussion 102. doi: 10.1227/01.neu.0000429842.61213.d5.

[17] Todeschi J, Bund C, Cebula H, Chibbaro S, Lhermitte B, Pin Y, Lefebvre F, Izzie JN, Proust F. Diagnostic value of fusion of the metabolic and structural images for stereotactic biopsy of brain tumors without enhancement after contrast medium injection. Neurochirurgie. 2019 Sep 24. pii: S0028-3770 (19) 30218-8. doi: 10.1016/j.neuchi.2019.08.002. [Epub ahead of print] PubMed PMID: 31560911.

[18] Khan IS, Kelly PD, Singer RJ. Prototyping of cerebral vasculature physical models. Surg Neurol Int. 2014 Jan 27; 5: 11. doi: 10.4103/2152-7806.125858. eCollection 2014. PubMed PMID: 24678427; PubMed Central PMCID: PMC3942610.

[19] Qiu K, Haghiashtiani G, McAlpine MC. 3D Printed Organ Models for Surgical Applications. Annu Rev Anal Chem (Palo Alto Calif). 2018 Jun 12; 11 (1): 287-306. doi: 10.1146/annurev-anchem-061417-125935. Epub 2018 Mar 28. Review. PubMed PMID: 29589961; PubMed Central PMCID: PMC6082023.

[20] Brown C, Robinson D, Egan R, Hopkins L, Abdelrahman T, Powell A, Pollitt MJ, Lewis WG. Prospective Cohort Study of Haptic Virtual Reality Laparoscopic Appendicectomy Learning Curve Trajectory. J Laparoendosc Adv Surg Tech A. 2019 Sep; 29 (9): 1128-1134. doi: 10.1089/lap.2019.0332. Epub 2019 Jul 30. PubMed PMID: 31361560.

[21] Xu Y, Tian W, Wei Z, Li Y, Gao X, Li W, Dong B. Microcatheter shaping using three-dimensional printed models for intracranial aneurysm coiling. J Neurointerv Surg. 2019 Sep 28. pii: neurintsurg-2019-015346. doi: 10.1136/neurintsurg-2019-015346. [Epub ahead of print] PubMed PMID: 31563890.

[22] Nagassa RG, McMenamin PG, Adams JW, Quayle MR, Rosenfeld JV. Advanced 3D printed model of middle cerebral artery aneurysms for neurosurgery simulation. 3D Print Med. 2019 Aug 1; 5 (1): 11. doi: 10.1186/s41205-019-0048-9. PubMed PMID: 31372773; PubMed Central PMCID: PMC6743137.

[23] Kim PS, Choi CH, Han IH, Lee JH, Choi HJ, Lee JI. Obtaining Informed Consent Using Patient Specific 3D Printing Cerebral Aneurysm Model. J Korean Neurosurg Soc. 2019 Jul; 62 (4): 398-404. doi: 10.3340/jkns.2019.0092. Epub 2019 Jul 1. PubMed PMID: 31290295; PubMed Central PMCID: PMC6616983.

[24] Levitt MR, Mandrycky C, Abel A, Kelly CM, Levy S, Chivukula VK, Zheng Y, Aliseda A, Kim LJ. Genetic correlates of wall shear stress in a patient-specific 3D-printed cerebral aneurysm model. J Neurointerv Surg. 2019 Oct; 11 (10): 999-1003. doi: 10.1136/neurintsurg-2018-014669. Epub 2019 Apr 12. PubMed PMID: 30979845; PubMed Central PMCID: PMC6744304.

[25] Bairamian D, Liu S, Eftekhar B. Virtual Reality Angiogram vs 3-Dimensional Printed Angiogram as an Educational tool-A Comparative Study. Neurosurgery. 2019 Aug 1; 85 (2): E343E349. doi: 10.1093/neuros/nyz003. PubMed PMID: 30715444

[26] Sullivan S, Aguilar-Salinas P, Santos R, Beier AD, Hanel RA. Three-dimensional printing and neuroendovascular simulation for the treatment of a pediatric intracranial aneurysm: case report. J Neurosurg Pediatr. 2018 Dec 1; 22 (6): 672-677. doi: 10.3171/2018.6. PEDS17696. PubMed PMID: 30215588.

[27] Ruedinger KL, Rutkowski DR, Schafer S, Roldán-Alzate A, Oberstar EL, Strother C. Impact of image reconstruction parameters when using 3D DSA reconstructions to measure intracranial aneurysms. J Neurointerv Surg. 2018 Mar; 10 (3): 285-289. doi: 10.1136/neurintsurg-2017-013080. Epub 2017 Apr 6. PubMed PMID: 28385725. 
[28] Benet A, Plata-Bello J, Abla AA, Acevedo-Bolton G, Saloner D, Lawton MT. Implantation of 3D-Printed Patient-Specific Aneurysm Models into Cadaveric Specimens: A New Training Paradigm to Allow for Improvements in Cerebrovascular Surgery and Research. Biomed Res Int. 2015; 2015: 939387. doi: 10.1155/2015/939387. Epub 2015 Oct 11.
PubMed PMID: 26539542; PubMed Central PMCID: PMC4619899.

[29] Michael T. Lawton, MD, and Michael J. Lang, MD. The future of open vascular neurosurgery, perspectives on cavernous malformations, AVMs, and bypasses for complex aneurysms. JNSPG $7^{\text {th }}$ Anniversary Invited Review Article. 